\title{
Soliton laser: A computational two-cavity model
}

\author{
Berg, P.; If, F.; Christiansen, Peter Leth; Skovgaard, Ove
}

Published in:

Physical Review A

Link to article, DOI:

10.1103/PhysRevA.35.4167

Publication date:

1987

Document Version

Publisher's PDF, also known as Version of record

Link back to DTU Orbit

Citation (APA):

Berg, P., If, F., Christiansen, P. L., \& Skovgaard, O. (1987). Soliton laser: A computational two-cavity model. Physical Review A, 35(10), 4167-4174. https://doi.org/10.1103/PhysRevA.35.4167

\section{General rights}

Copyright and moral rights for the publications made accessible in the public portal are retained by the authors and/or other copyright owners and it is a condition of accessing publications that users recognise and abide by the legal requirements associated with these rights.

- Users may download and print one copy of any publication from the public portal for the purpose of private study or research.

- You may not further distribute the material or use it for any profit-making activity or commercial gain

- You may freely distribute the URL identifying the publication in the public portal

If you believe that this document breaches copyright please contact us providing details, and we will remove access to the work immediately and investigate your claim 


\title{
Soliton laser: A computational two-cavity model
}

\author{
P. Berg, F. If, P. L. Christiansen, and O. Skovgaard \\ Laboratory of Applied Mathematical Physics, The Technical University of Denmark, DK-2800 Lyngby, Denmark
}

(Received 7 November 1986)

\begin{abstract}
An improved computational two-cavity model of the soliton laser proposed and designed by Mollenauer and Stolen [Opt. Lett. 9, 13 (1984)] is obtained through refinements of (i) the laser cavity model, (ii) the pulse propagation in the fiber cavity, and (iii) the coupling between the two cavities. As a result of the coupling to the fiber cavity, stable output pulses of temporal width 7.5 psec from the laser cavity are narrowed by a factor of approximately 10 .
\end{abstract}

\section{INTRODUCTION}

In 1980, Mollenauer, Stolen, and Gordon ${ }^{1}$ observed picosecond pulse narrowing of solitons in optical fibers experimentally. This observation was used in the soliton laser proposed and designed by Mollenauer and Stolen ${ }^{2,3}$ in 1984. It was demonstrated experimentally that this device could produce stable pulses of width $210 \mathrm{fsec}$ and later ${ }^{4}$ of width down to $100 \mathrm{fsec}$. Compression in a second, external fiber has further reduced these pulse widths to less than $50 \mathrm{fsec}$, and a reduction by at least another factor of 2 is considered likely in the near future. ${ }^{3}$ As illustrated in Fig. 1, the soliton laser is a double-cavity system consisting of a synchronously pumped, modelocked color-center laser ${ }^{5}$ coupled to an optical singlemode fiber acting as a control cavity.

An analytical theory based on a single-cavity model of the soliton laser was proposed by Haus and Islam. ${ }^{6}$ Experimental results, ${ }^{4}$ however, indicate that the two-cavity soliton laser cannot be reduced to an equivalent single cavity.

A simple computational model presented by If et al. $^{7}$ was able to reproduce the essential features of the soliton laser dynamics. However, only quasistability of the pulses and a small pulse-narrowing effect was obtained.

The numerical simulation by Blow and $\mathrm{Wood}^{8}$ predicts the expected final two-soliton state. Here the solitons have different velocities, giving rise to double-peaked output pulses from the system.

In the present work the model used by If et al..$^{7}$ is improved by (i) a more sophisticated model of the colorcenter laser by introduction of saturation through a time-

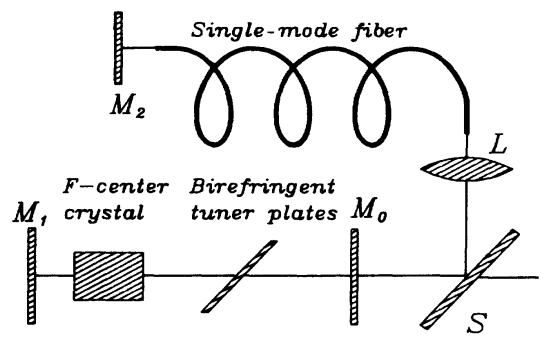

FIG. 1. Schematic diagram of the soliton laser. dependent inversion population, (ii) effective separation of neighboring pulses on the fiber through introduction of absorbing boundary layers in the numerical scheme, and (iii) correct description of the coupling between the two cavities in the soliton-laser system.

The paper is structured as follows. Section II contains a detailed description of the laser cavity and the numerical treatment of the cavity modes. In Sec. III pulse propagation in the fiber modeled by a loss-perturbed nonlinear Schrödinger equation is treated by perturbation theory and an improved version of the split-step Fourier method. Section IV contains our numerical results for the combined system, which finally are discussed in Sec. V.

\section{COLOR-CENTER LASER}

The laser cavity shown in Fig. 1 formed by the mirrors $M_{0}$ and $M_{1}$ consists of a lasing medium (an $F$-center crystal) and a pair of birefringent tuner plates. ${ }^{9}$ The laser is mode-locked by synchronous pumping through the mirror $M_{1}$ from a Nd:YAG laser (where YAG represents yttrium aluminum garnet). The pumping frequency $\omega_{M}$, tuned to match the cavity round trip time $T_{c}$, locks regions of the large laser bandwidth onto different polarization directions. The birefringent tuner plates operate as a bandpass filter restricting the bandwidth to one region with a single polarization direction. This allows us to use the scalar version of the Maxwell-Bloch equations ${ }^{10}$ as the model equations for the laser cavity:

$$
\begin{aligned}
& c \mathscr{C}_{z}+\dot{\mathscr{C}}=i \frac{\omega_{21}}{2 \epsilon_{0}} \mathscr{P}-\kappa \mathscr{E}, \\
& \dot{\mathscr{P}}=-\left(i \Delta+\gamma_{\perp}\right) \mathscr{P}+\frac{1}{i \hbar} M \mathscr{D} \mathscr{E}, \\
& \dot{\mathscr{D}}=\gamma_{\|}\left(\mathscr{D}_{0}-\mathscr{D}\right)+\frac{2}{i \hbar}\left(\mathscr{C} \mathscr{P}^{*}-\mathscr{C}^{*} \mathscr{P}\right),
\end{aligned}
$$

where the subscript $z$ denotes differentiation with respect to $z$, and the dots denote temporal differentiation. Equation (2.1a) describes the one-way pulse propagation of the complex electric field envelope $\mathscr{E}(z, t)$ in the cavity. Here the vacuum light velocity $c=\left(\mu_{0} \epsilon_{0}\right)^{-1 / 2}, \kappa$ is the cavity decay rate, and $\omega_{21}$ is the atomic transition frequency. In Eq. (2.1b), $\Delta=\omega_{21}-\omega_{0}$ is the detuning of the laser from an appropriate reference carrier frequency $\omega_{0}$. 
The polarization $\mathscr{P}$, which acts as a source for the electric field, is governed by Eq. (2.1b). The bandwidth $\gamma_{\perp}$ of the color-center laser (CCL) is very large compared with the signal bandwidth. ${ }^{3}$ Therefore the polarization is forced to follow the electric field, i.e., $\dot{\mathscr{P}} \simeq 0$ (the rate equation approximation ${ }^{11}$ ). Since the laser operates at a frequency near $\omega_{21}$, the detuning $\Delta$ is small compared with the laser bandwidth $\gamma_{\perp}$. Therefore the factor $-\left(i \Delta+\gamma_{\perp}\right)$ is approximated by $-\gamma_{\perp}$ in Eq. (2.1b). As a consequence, dispersion effects are ignored in this model of the CCL. Finally, Eq. (2.1c) describes the evolution of the inversion population $\mathscr{D}$. The relaxation to its equilibrium value $\mathscr{D}_{0}$, produced by spontaneous emission, is described by the longitudinal relaxation constant $\gamma_{\|}$.

Shifting to normalized variables $t / T \rightarrow t, z / c T \rightarrow z$, $E=\mathscr{C} / \mathscr{C}_{0}$, and $D=\mathscr{D} / \mathscr{D}_{0}$ (see Secs. III and IV for numerical values), we then reduce Eqs. (2.1a) - (2.1c) to

$$
\begin{aligned}
& E_{z}+\dot{E}=\left(\alpha D-\kappa^{\prime}\right) E, \\
& \dot{D}=\gamma_{\|}^{\prime}-\gamma_{\|}^{\prime}\left(1+|E|^{2} / I_{s}\right) D .
\end{aligned}
$$

Here, $\kappa^{\prime}=T \kappa, \gamma_{\|}^{\prime}=T \gamma_{\|}$. The gain $\alpha=T \omega_{21} M \mathscr{D}{ }_{0} / 2 \epsilon_{0} \hbar \gamma_{\perp}$ and the saturation $I_{s}=\gamma_{\perp} \gamma_{\|} \hbar^{2} / M \mathscr{C}_{0}^{2}$ will be used as model parameters (see Fig. 2). As seen from Eq. (2.2b) the system will saturate at a certain field intensity through a speedup of the relaxation of the inversion to its equilibrium value.

In an earlier model $^{7}$ of the CCL, the system saturation was included phenomenologically in the gain parameter as $\alpha^{\prime}=\alpha \exp \left(-W / W_{s}\right)$, with $W$ being the total pulse energy in the cavity and $W_{s}$ its saturation energy.

In the limit of low intensity, $\dot{D} \simeq 0$ in Eq. (2.2b), we obtain

$$
\begin{aligned}
& E_{z}+\dot{E}=\left(\alpha^{\prime}-\kappa^{\prime}\right) E, \\
& \alpha^{\prime}=\alpha\left(1+|E|^{2} / I_{s}\right)^{-1} .
\end{aligned}
$$

For small energies the $\alpha^{\prime}$ used in the laser model in Ref. 7 is obtained by replacing $|E|^{2} / I_{s}$ by $W / W_{s}$.

The synchronized pumped CCL is modeled as an actively mode-locked system ${ }^{11}$ by introducing a modulated cavity decay rate

$$
\kappa^{\prime}=\kappa_{0}+A_{M}\left\{1-\cos \left[\omega_{M}(z-t)\right]\right\} .
$$

Here, $\omega_{M}=2 \pi / L_{c}$, where $L_{c}$ is the normalized unfolded cavity length.

The action of the birefringent tuner plates is to reduce the signal bandwidth of the CCL. The center frequency is equal to the center frequency of the $\mathrm{CCL}, \omega_{0}$, and the bandwidth $\Omega_{p} \ll<\gamma_{\perp}$. In the frequency domain we write the transfer function of the plates as

$$
B(\omega)=\frac{1-q_{0}}{1+\left[\left(\omega-\omega_{0}\right) / \Omega_{p}\right]^{2}}+q_{0},
$$

ignoring possible dispersive effects. The parameter $q_{0}$, the minimum of the transfer function, depends on the material and the number of plates.

Equations (2.2a) and (2.2b) with Eq. (2.4) are solved numerically by expanding the electric field in $2 N+1$ cavity modes with mode spacing $\Delta \omega=2 \pi / T_{c}$

$$
E(z, t) \simeq \sum_{n=-N}^{N} E_{n}(t) \exp [-i n \Delta \omega(z-t)] .
$$

Approximating the field intensity with its spatial average, $I(t)=\left\langle|E(z, t)|^{2}\right\rangle$, we obtain a system of coupled ordinary differential equations for the normalized cavity modes and inversion

$$
\begin{aligned}
& \dot{E}_{n}=\left(\alpha D-\kappa_{0}\right) E_{n}+\beta_{n}\left(E_{n+1}-2 E_{n}+E_{n-1}\right), \\
& \dot{D}=\gamma_{\|}^{\prime}-\gamma_{\|}^{\prime}\left[1+I(t) / I_{s}\right] D .
\end{aligned}
$$

The bandwidth $\Omega_{c}$ of the effective mode coupling is accomplished in a phenomenological way by

$$
\beta_{n}=\frac{1}{2} A_{M} e^{-\left(n \Delta \omega / \Omega_{c}\right)^{2} / 2},
$$

and the field intensity is calculated as the sum $I(t) \simeq \sum_{n=-N}^{N}\left|E_{n}(t)\right|^{2}$.
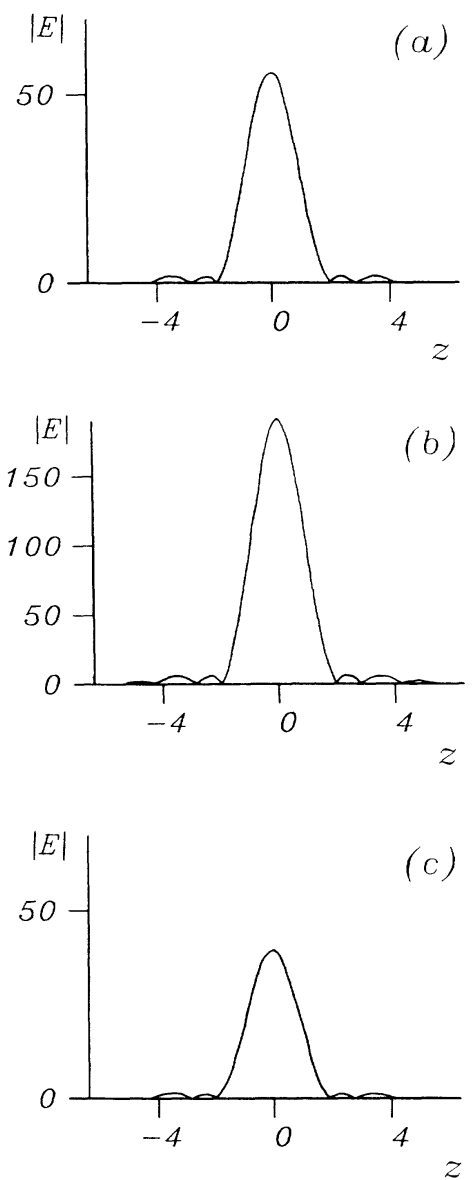

FIG. 2. Stable pulses produced in the laser cavity from spatial noise after 75 cavity round-trip times. Parameters: $R_{0}=83 \%, \quad \kappa_{0}=0.2454, \quad \gamma_{\|}^{\prime}=0.1227, \quad \Omega_{c}=1.473, \quad A_{M}=2$, $q_{0}=0.5, \quad \Omega_{p}=2.454$; (a) $\alpha=0.4909, \quad I_{s}=420$, (b) $\alpha=2.454$, $I_{s}=420$, (c) $\alpha=0.4909, I_{s}=210$. With the time normalization constant $T=10 \mathrm{psec}$, these pulses correspond to temporal output pulses from the laser cavity of a full width at half maximum (FWHM) of $7.5 \mathrm{psec}$. 
The cavity modes are advanced in time according to Eqs. (2.7a) and (2.7b) by using a forward finite difference approximation to the time derivatives. ${ }^{12}$ This scheme is convergent and absolutely stable, if the time step $\Delta t$ satisfies

$$
\Delta t<\min \left[2 /\left(\kappa_{0}-\alpha D\right), 2 /\left(\gamma_{\|}^{\prime}+\gamma_{\|}^{\prime} I / I_{s}\right)\right] .
$$

In Fig. 2 we show the stable pulses, $\left|E\left(z, 75 T_{c}\right)\right|$, produced in the laser cavity for different value of the gain parameter $\alpha$ and the saturation parameter $I_{s}$. In all cases, stable pulses result from initial spatial noise, after 75cavity round-trip times. The temporal output from the CCL cavity at the mirror $M_{0}$ (reflectivity $R_{0}<100 \%$ ), $z=L_{c}$, can be calculated as

$$
E\left(L_{c}, t\right)=\left(1-R_{0}^{2}\right)^{1 / 2} \sum_{n=-N}^{N} E_{n}(t) \exp (\text { in } \Delta \omega t)
$$

according to Eq. (2.6).

\section{OPTICAL FIBER}

\section{A. Perturbed nonlinear Schrödinger equation}

The appropriate evolution equation for optical pulse propagation within the fiber is the perturbed nonlinear Schrödinger equation ${ }^{13}$ (PNLS):

$$
i E_{z}+\frac{1}{2} E_{t t}+|E|^{2} E+i \gamma E=0 .
$$

Let $\mathscr{C}(z, t)=\phi(z, t) \exp \left[i\left(k_{0} z-\omega_{0} t\right)\right]$ be the electric field in the fiber, where $z$ is the coordinate along the fiber, $t$ is time, and $\phi(z, t)$ is a slowly varying envelope function. The linear propagation constant is expressed by the truncated expansion $k(\omega)=k_{0}+k_{1}\left(\omega-\omega_{0}\right)+\frac{1}{2} k_{2}\left(\omega-\omega_{0}\right)^{2}$; $k_{0}=k\left(\omega_{0}\right)$ is the propagation constant at the carrier frequency $\omega_{0}, k_{1}=\partial k / \partial \omega$ is the reciprocal group velocity $v_{g}^{-1}$, and $k_{2}=\partial^{2} k / \partial \omega^{2}=\left(\lambda_{0} / \omega_{0}\right) d_{v_{g}}$, where $\lambda_{0}$ is the vacuum wavelength at $\omega_{0}$ and $d_{v_{g}}=-d\left(v_{g}{ }^{-1}\right) / d \lambda$ is the group velocity dispersion. The silica single-mode fiber has minimal loss at $\lambda_{0} \simeq 1.5 \mu \mathrm{m}$, corresponding to negative $d_{v_{g}}$ and anomalous dispersion. ${ }^{3}$ Introducing the normalized variables $T^{-2}\left|k_{2}\right| z \rightarrow z, T^{-1}\left(t-z / v_{g}\right) \rightarrow t$, and $T\left(\rho /\left|k_{2}\right|\right)^{1 / 2} \phi(z, t) \rightarrow E(z, t)$, where $\rho=\frac{1}{2} k_{0} n_{0} / n_{2}, n_{2}$ is the Kerr coefficient in the expression $n_{0}(\omega)+n_{2}|\mathscr{E}|^{2}$ for the refractive index, and $T$ is a time scaling parameter, we get Eq. (3.1) for the normalized field, $E$. The coefficient $\gamma$ in the loss term $i \gamma E$ is given by $\gamma=T^{2} v_{0} /\left|k_{2}\right|$, where $v_{0}$ is the linear absorption along the fiber. Note that the normalized variable $t$ is delayed with velocity $v_{g}$ through a Galilei transformation. The arbitrary time scaling $T$ will be fixed in the following section.

An example of the relations between the dimensionless quantities and the corresponding physical variables for a typical fiber is given in Table I. For short fibers of low loss the term $i \gamma E$ in Eq. (3.1) can be neglected yielding the nonlinear Schrödinger equations (NLS) for $E(z, t)$.

\section{B. Solutions to the nonlinear Schrödinger equation}

Analytical solutions to the NLS are available to us through the original work by Zakharov and Shabat, ${ }^{14}$ who
TABLE I. Typical fiber data. ${ }^{1}$ With these data we have $\gamma=T^{2} 1.13 \times 10^{21} \quad \mathrm{sec}^{-2}, \quad T \phi 1.09 \times 10^{5} \quad \mathrm{~m} /(\mathrm{sec} \mathrm{V}) \rightarrow E$, $T^{-1}\left(t-z 4.83 \times 10^{-2} \mathrm{sec} / \mathrm{m}\right) \rightarrow t, T^{-2} z 20.4 \times 10^{-27} \mathrm{sec}^{2} / m \rightarrow z$, with $T$ being the time normalization parameter.

\begin{tabular}{ccccc}
\hline $\begin{array}{c}\lambda_{0} \\
(\mu \mathrm{m})\end{array}$ & $n_{0}$ & $\begin{array}{c}n_{2} \\
(\mathrm{~m} / \mathrm{V})^{2}\end{array}$ & $\begin{array}{c}d_{v_{\mathrm{g}}} \\
{[(\mathrm{psec} / \mathrm{nm}) / \mathrm{km}]}\end{array}$ & $\begin{array}{c}v_{0} \\
(\mathrm{~dB} / \mathrm{km})\end{array}$ \\
\hline 1.55 & 1.45 & $1.2 \cdot 10^{-22}$ & -16 & 0.2 \\
\hline \hline
\end{tabular}

showed that NLS possesses soliton solutions. In particular, Satsuma and Yajima ${ }^{15}$ have investigated initial-value problems for the NLS, using the inverse scattering transform. Initial pulses of the form

$$
E(0, t)=A \operatorname{sech}(t), \quad A>0
$$

produce $N$ discrete eigenvalues in the scattering problem, $N$ being an integer satisfying $|N-A|<\frac{1}{2}$. The eigenvalues are purely imaginary and given by

$$
\zeta_{k}=i \eta_{k}=i\left(A-k+\frac{1}{2}\right), \quad k=1, \ldots, N .
$$

This is called the $N$-soliton case, and for Eqs. (3.1) and (3.2) with $\gamma=0$ and $A=N$ analytical $N$-soliton solutions exist. For noninteger $A$ the solution consists of $N$ solitons and radiation. ${ }^{15}$ The radiation is "peeled off" the pulse during the propagation on the fiber. It is important to note that the $N$ solitons $(N \geqq 2)$ are deeply modulated pulses which are periodic in $z$ except for a constant phase shift, returning to the same profile given by Eq. (3.2) for distances equal to multiples of the soliton period ${ }^{15}$

$$
z_{0}=\frac{\pi}{2}
$$

The results also hold for initial pulses of width $T_{0}$ and amplitude $E_{0}$

$$
E(0, t)=E_{0} \operatorname{sech}\left(t / T_{0}\right),
$$

provided that $E_{0}=A / T_{0}$, owing to the scaling properties of NLS. For $\mu$ real, the NLS is invariant under the scaling

$$
s=t / \mu, \quad \xi=z / \mu^{2}, \quad e(\xi, s)=\mu \mathrm{E}(z, t) .
$$

Thus the soliton period resulting from initial pulse (3.5) with $E_{0} T_{0}=N \geqq 2$ is given by

$$
z_{0}=\frac{\pi}{2} T_{0}^{2} \text {. }
$$

Because $z_{0}$ scales with $T_{0}^{2}$, a broader pulse requires a longer distance in the fiber to complete one period, and to a given fiber length there corresponds a pulse of definite width which goes through one period between the fiber ends. In other words, if the periodicity length of the pulse can be shortened then the pulse is temporally narrowed. In Fig. 3, the evolution of the two-soliton solution, $E(0, t)=2 \operatorname{sech}(t)$, through one period is shown. The overall phase shift is $\pi / 4$ for the two-soliton solution.

If the pulse differs slightly from the exact two-soliton solution the "period" is also changed. The term "period" refers to the soliton part of the solution. With perturbed 


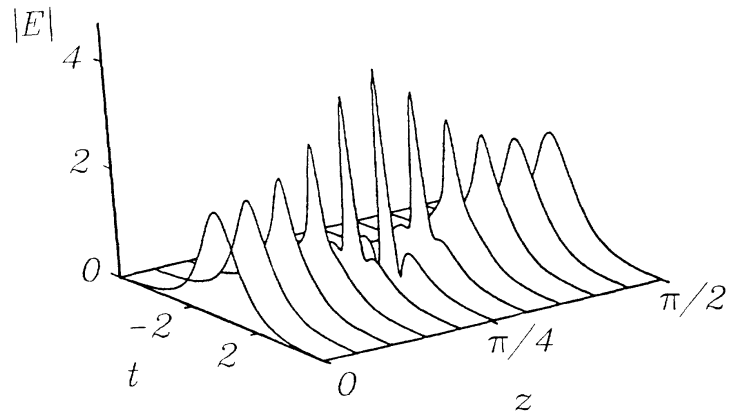

FIG. 3. Propagation of the two-soliton solution on a fiber of length equal to one soliton period. $|E|=|E(z, t)|$ is shown.

initial condition the solution to NLS is not strictly periodic. This we explain by means of perturbation theory: Assume that the $E=2 \operatorname{sech}(t)$ undergoes a small perturbation:

$$
2 \operatorname{sech}(t) \rightarrow 2(1+\epsilon) \operatorname{sech}[t /(1+\delta)] .
$$

Using the scalings (3.6) with $\mu=(1+\delta)$ we obtain from Eq. (3.3) the shift in both eigenvalues $\Delta \eta_{1}=\Delta \eta_{2}$ $=2(\epsilon+\delta+\epsilon \delta)$. Since the soliton period is related to the eigenvalues through ${ }^{15}$

$$
z_{0}=\frac{\pi}{\eta_{1}^{2}-\eta_{2}^{2}}
$$

the corresponding period in $z$ becomes

$$
z_{0}(\epsilon, \delta)=z_{0} \frac{(1+\delta)^{2}}{1+2(\epsilon+\delta+\epsilon \delta)}
$$

where $z_{0}$ is the unperturbed period given by Eq. (3.4). From Eq. (3.10) we see that for $\delta=0$ the period is either extended or contracted, depending on whether $\epsilon<0$ or $\epsilon>0$, while for $\epsilon=0$ every change in pulse width extends the period, since $z_{0}(0, \delta)$ has minimum for $\delta=0$. If the input pulse on the fiber has a slightly too high amplitude (and therefore a period which is too short to match the exact two-soliton period) the period may be changed to the required value of $z_{0}$ simply by decreasing the width of the pulse by an amount $\delta$ found by equating $z_{0}(\epsilon, \delta)$ with $z_{0}$ in Eq. (3.10). Thus

$$
\frac{\delta^{2}}{1+\delta}=2 \epsilon
$$

Note that in order to maintain the existence of the twosoliton solution the values of $\epsilon$ and $\delta$ must satisfy $|\epsilon+\delta+\epsilon \delta|<\frac{1}{4}$. On the other hand, if the initial pulse has too low amplitude, i.e., $\epsilon<0$, the period cannot be changed to $z_{0}$, since this requires $\delta<-1$.

In Fig. 4, we compare numerical solutions of NLS obtained for the exact two-soliton and for a perturbed twosoliton case satisfying Eq. (3.11) as initial conditions.

\section{Split-step Fourier method for the perturbed nonlinear Schrödinger equation}

Consider the PNLS (3.1) with boundary-initial conditions
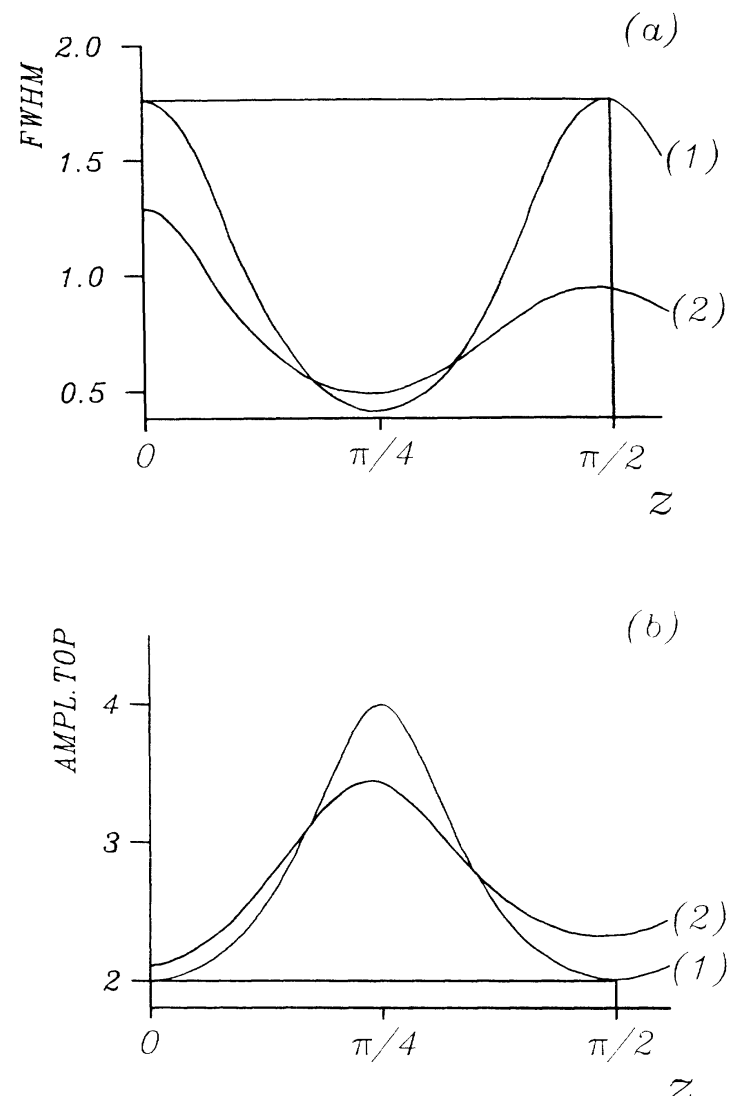

FIG. 4. Pulse width, FWHM, (a) and maximum amplitude, AMPL.TOP, (b), along the fiber for initial pulses $E(0, t)=2(1+\varepsilon) \operatorname{sech}[t /(1+\delta)] . \quad$ (1) $\varepsilon=\delta=0, \quad$ (2) $\varepsilon=0.05$, $\delta=-0.2702$.

$$
\begin{aligned}
& E(z, t) \rightarrow 0, \quad E_{t}(z, t) \rightarrow 0 \text { as } t \rightarrow \pm \infty, \\
& E(0, t)=E_{0}(t) .
\end{aligned}
$$

In Eq. (3.1), $\gamma$ is assumed real and independent of $z$. The equation can be separated into a linear part

$$
i \widehat{E}_{z}+\frac{1}{2} \widehat{E}_{t t}=0
$$

and a nonlinear part

$$
i \widetilde{E}_{z}+|\widetilde{E}|^{2} \widetilde{E}+i \gamma \widetilde{E}=0 .
$$

Equations (3.13) and (3.14) can both be solved exactly. The solution of Eq. (3.13) becomes

$$
F(z, \omega)=F(0, \omega) \exp \left(-i \frac{1}{2} \omega^{2} z\right)
$$

in Fourier space. For Eq. (3.14) we easily derive $\left(|\widetilde{E}|^{2}\right)_{z}=-2 \gamma|\widetilde{E}|^{2}$, with the solution

$$
|\widetilde{E}(z, t)|^{2}=|\widetilde{E}(0, t)|^{2} e^{-2 \gamma z} .
$$

Insertion of the expression (3.16) into (3.14) yields

$$
\widetilde{E}_{z}=\left[i|\widetilde{E}(0, t)|^{2} e^{-2 \gamma z}-\gamma\right] \widetilde{E},
$$

from which we find the exact solution of Eq. (3.14) 


$$
\widetilde{E}(z, t)=\widetilde{E}(0, t) \exp \left[i|\widetilde{E}(0, t)|^{2}\left(1-e^{-2 \gamma z}\right) / 2 \gamma-\gamma z\right] .
$$

In our extension of the split-step Fourier method ${ }^{16,17}$ the solution to Eq. (3.1) is advanced one space step $\Delta z$ by (i) obtaining $E(\Delta z, t)$ from $E(0, t)$ by means of (3.17), (ii) inserting the Fourier transform of $E(\Delta z, t)$ as $F(0, \omega)$ in (3.15), and (iii) transforming the resulting $F(\Delta z, \omega)$ back to $t$ space. For numerical solution of the linear equation, fast Fourier transform (FFT) is used, which requires periodic boundary conditions. By choosing the time interval $T_{B}$ sufficiently large, (3.12a) is replaced by $E\left(z,-T_{B} / 2\right)=E\left(z, T_{B} / 2\right)=0$, thus gaining the necessary periodicity. This method is accurate to second-order in $\Delta z$ and all orders in $\Delta t$ and is unconditionally stable. ${ }^{16}$

Clearly, the scheme also holds if $\gamma$ is a function of $t$. If we let

$$
\gamma(t)=\gamma_{0}\left\{\operatorname{sech}^{2}\left[\alpha\left(t-T_{B} / 2\right)\right]+\operatorname{sech}^{2}\left[\alpha\left(t+T_{b} / 2\right)\right]\right\},
$$

where $\gamma_{0}$ and $\alpha$ are positive constants, smooth losses are introduced in layers at the boundaries, see Fig. 5. This choice of $\gamma$ has the effect of absorbing outgoing radiation $^{18}$ without violating the periodicity requirement of the Fourier method. The parameters $\gamma_{0}$ and $\alpha$ can be chosen such that scattering from the "absorption walls" is small over the range of frequencies used in the FFT, see Appendix.

The effect of the absorbing boundary layers is demonstrated in Fig. 6. For simplicity, an example of a onesoliton solution with radiation superposed is chosen as initial condition

$$
E(0, t)=[1+0.6 \cos (7 t)] \operatorname{sech}(t) .
$$

In the case of no absorption, Fig. 6(a), the radiation cannot escape the system and eventually destroys the onesoliton solution, while, if the $\gamma$ term (3.18) is included, the radiation is essentially absorbed at the first passage of the boundary layer, leaving the one-soliton solution undisturbed, Fig. 6(b).

By introducing the $\gamma$ loss in our model we avoid interaction between neighboring pulses. Such interaction does not occur in the physical system because of its long repetition period compared with the pulse width.

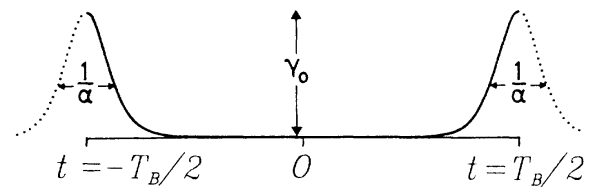

FIG. 5. Absorption function $\gamma(t)$ given by Eq. (3.18) introduces lossy boundary layers at the periodic boundaries $t= \pm T_{B} / 2$. Parameters $\gamma_{0}$ and $\alpha$ in Eq. (3.18) must be chosen such that the scattering from the "absorption walls," $\gamma_{0} \operatorname{sech}^{2}\left[\alpha\left(t \mp T_{B} / 2\right)\right]$, is small.
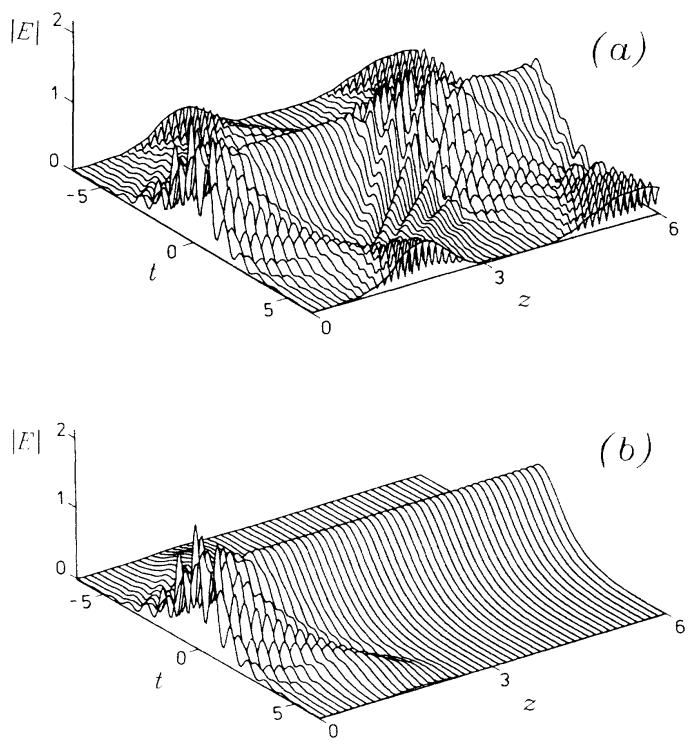

FIG. 6. Evolution of the one-soliton plus radiation solution, Eq. (3.19), with dynamics given by (a) classical NLS, Eq. (3.1) with $\gamma \equiv 0$, (b) NLS with absorption, Eqs. (3.1) and (3.18). Parameters: $T_{B}=12.8, \quad \gamma_{0}=20, \quad \alpha=1$. Resolution $\Delta t=0.1$, $\Delta z=0.005$.

\section{DOUBLE-CAVITY SYSTEM}

As shown in Fig. 1 the soliton laser is a double-cavity system, consisting of a CCL cavity bounded by the mirrors $M_{1}$ (reflectivity $R_{1} \sim 100 \%$ ) and $M_{0}$ (reflectivity $R_{0}<100 \%$ ), and a fiber cavity bounded by $M_{0}$ and the movable mirror $M_{2}$ (reflectivity $R_{2} \sim 100 \%$ ). The lens $L$ focuses the pulse launch into the fiber (transmission coefficient $T_{L}$ ). The output from the cavity system is through the beam splitter $S$ (reflectivity $R_{S}<100 \%$ ).

In Fig. 7 the pulses propagating into the laser and out of the laser are denoted $E_{T L}$ and $E_{F L}$, respectively. Similarly, pulses into the fiber and out of the fiber are denoted $E_{T F}$ and $E_{F F}$, respectively. The coupling between the two cavities is approximated by the equations

$$
\begin{aligned}
& E_{T L}=R_{0} E_{F L}+\left(1-R_{0}^{2}\right)^{1 / 2} R_{s}\left(e^{i \phi} E_{F F}\right), \\
& E_{T F}=\left(1-R_{0}^{2}\right)^{1 / 2} R_{S} T_{L} E_{F L}+R_{S}^{2} R_{0} T_{L}\left(e^{i \phi} E_{F F}\right) .
\end{aligned}
$$

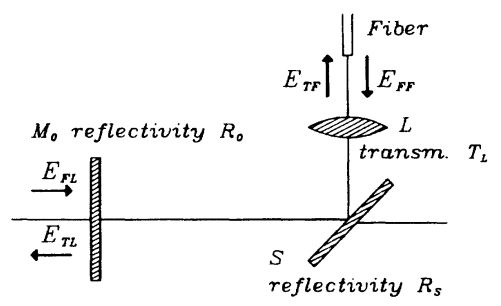

FIG. 7. Detailed diagram of the coupling section between the CCL cavity and the fiber cavity. Relations between the electric fields in the two cavities are given in Eq. (4.1). 
As expressed in Eq. (4.1a) $E_{T L}$ is a superposition of the partially reflected pulse from $M_{0}, R_{0} E_{F L}$, and the pulse from the fiber reflected by the beam splitter $S$ and transmitted through $M_{0},\left(1-R_{0}^{2}\right)^{1 / 2} R_{S} e^{i \phi} E_{F F}$. Here, $e^{i \phi}$ is an overall phase factor modeling small dynamical adjustments by the mirror $\boldsymbol{M}_{2}$ (shown in Fig. 1). As described in Ref. 4 this adjustment is needed in the experiment in order to ensure that the pulses $E_{F L}$ and $E_{F F}$ are superimposed in phase. The stability of the device turns out to depend on this phase adjustment. The same stability requirements are observed in our computational model.

In Eq. (4.1b) the pulse propagating into the fiber, $E_{T F}$, is written as a superposition of $E_{F L}$ transmitted through $M_{0}$, reflected at $S$, and focused by $L$, $\left(1-R_{0}^{2}\right)^{1 / 2} R_{S} T_{L} E_{F L}$, and $E_{F F}$ reflected at $S, M_{0}, S$ and focused by $L, R_{S} R_{0} R_{S} T_{L} e^{i \phi} E_{F F}$, where $e^{i \phi}$ is again the overall phase factor. The transmission coefficient $T_{L}$ approximates the focusing, leading to a two-soliton solution on the fiber with proper width and energy. The narrow beam coming out of the fiber goes through the lens with negligible loss. The spot sizes of the laser mode and of the fiber mode as seen on the mirror $M_{0}$ are not the same.

In Fig. 8 the results from a simulation with a fairly long fiber are shown. With the time normalization parameter $T=10 \mathrm{psec}$, chosen so that the laser emits pulses with realistic widths, the normalized fiber length in this simulation, $l=0.03125$, corresponds to a physical fiber length of $\sim 150 \mathrm{~m}$, see Table I. The CCL cavity emits stable pulses of temporal full width at half maximum (FWHM) of $7.5 \mathrm{psec}$. When the fiber cavity is connected, initially these broad pulses perform only a small fraction of a two-soliton oscillation on the fiber, returning with a much smaller temporal width [see Fig. 8(b)]. This pulse adds on to the next pulse from the CCL cavity (in phase due to the factor $e^{i \phi}$ ) to form a new pulse in the fiber with a smaller temporal width than that of the previous pulse. In Fig. 8(a) the system is seen to stabilize after approximately 100 cavity round trips (periods). Then the system emits stable pulses with a temporal width of 3.5 psec [see Fig. 8(c)]. According to Eq. (3.5) this width corresponds approximately to a full soliton oscillation on the fiber.

In Fig. 8(c) the propagation of the pulse along the fiber in the last period of the simulation is shown. The corresponding FWHM is plotted as a function of $z$ in Fig. 8(d), indicating that the stable operation of the soliton laser is a configuration where the two-soliton solution performs a slightly longer period of oscillation than the actual fiber length. This period of oscillation is shifted, giving rise to a pulse at exit from the fiber of width slightly smaller than the width of the input pulse. The interaction with the pulse from the CCL cavity then compensates for this discrepancy.

Simulations with shorter fiber lengths, $l=80 \mathrm{~m}$ and 40 $\mathrm{m}$, are shown in Figs. 9(a) and 9(b), respectively. The same qualitative behavior as in Fig. 8 is observed. As a result we obtain pulses of temporal widths (FWHM) approximately equal to 1.8 and $0.88 \mathrm{psec}$, respectively. Here, as elsewhere in the work, the accuracy of the numerical results was checked by doubling the number of modes (from 256 to 512) in Eq. (2.6) and, correspondingly, augmenting the space and time resolution used in the nu-
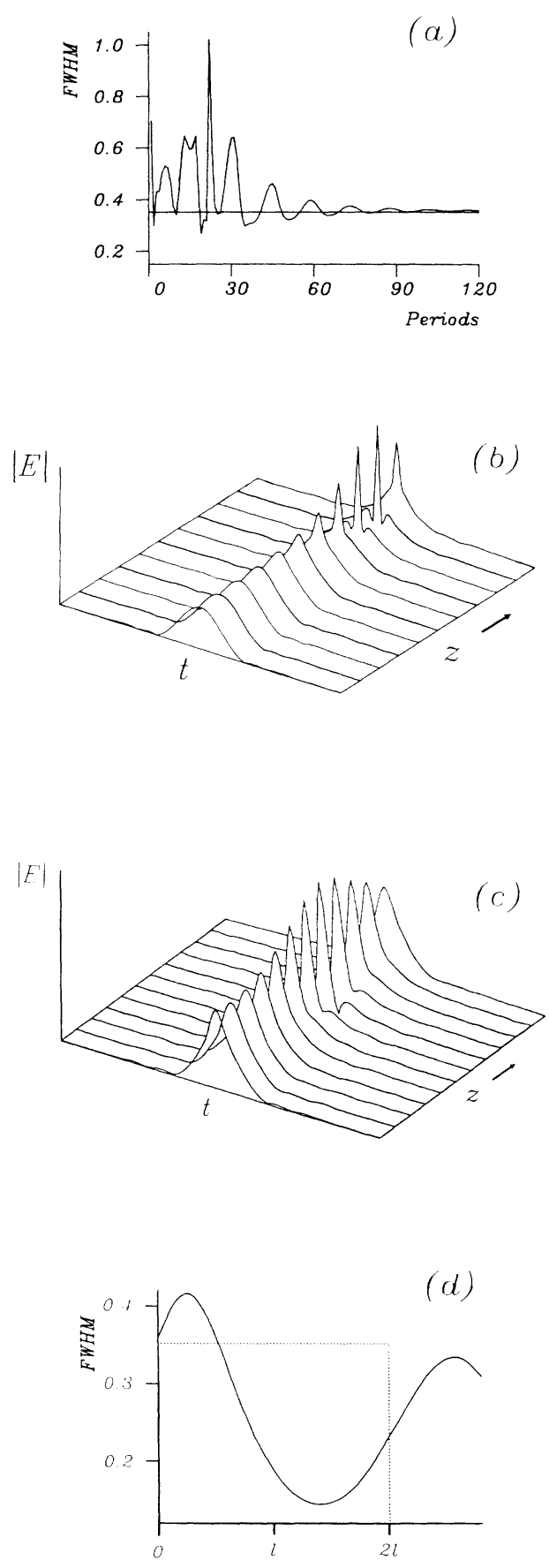

FIG. 8. Simulation with normalized fiber length $l=0.032$. $R_{0}=83 \%, R_{S}=65 \%, T_{L}=100 \%, \kappa_{0}=0.2454, \gamma^{\prime}=0.01473$, $\Omega_{c}=1.473, \quad A_{M}=2, \quad q_{0}=0.5, \quad \Omega_{p}=2.454, \quad \alpha=0.4909$, and $I_{s}=300$. (a) The normalized width (FWHM) of the pulses at output from the soliton laser versus cavity round-trip times (periods). The pulse width is compressed by the fiber to the theoretical width (indicated by horizontal line) (FWHM) 0.35 after 120 periods. (b) First propagation period of the pulse along the fiber. (c) Last propagation period (after 120 periods) of the pulse along the fiber, showing the typical two-soliton oscillation. (d) Normalized FWHM of the pulse versus fiber distance, after 120 periods. 

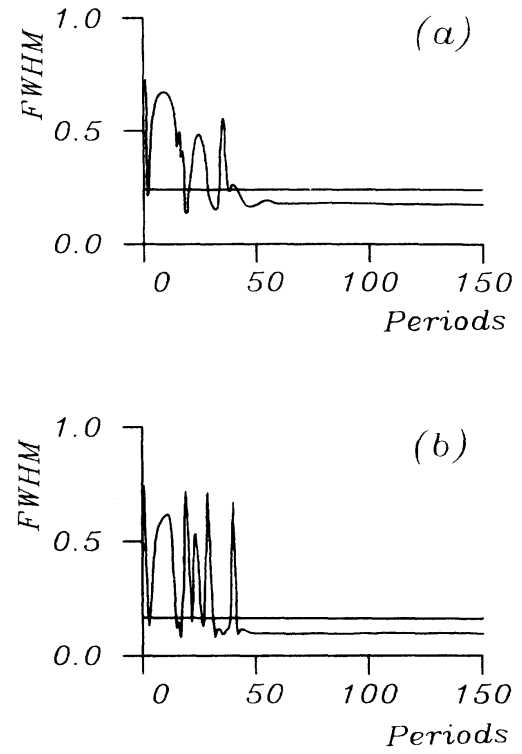

FIG. 9. Simulations showing the normalized width (FWHM) of output pulses versus cavity round-trip times (periods). Horizontal lines are theoretical pulse widths. Parameters, except $I_{s}$, $R_{S}$, and $T_{L}$ as in Fig. 8. (a) Fiber length $80 \mathrm{~m}$. $I_{s}=750$, $R_{S}=66 \%, T_{L}=71 \%$. Width of stable output pulses of approximately 1.8 psec. (b) Fiber length $40 \mathrm{~m} . I_{s}=750, R_{S}=66 \%$, $T_{L}=100 \%$. Width of stable output pulses of $0.88 \mathrm{psec}$.

merical schemes.

An attempt to produce pulse narrowings of a factor larger than 10 by application of even shorter fiber lengths failed because of unstable operation of the system. However, we believe that this difficulty could easily be overcome by including a larger number of cavity modes in the numerical calculations.

\section{DISCUSSION}

A genuine two-cavity numerical model of the Mollenauer-Stolen soliton laser is developed. In contrast to the earlier model presented by If et al., ${ }^{7}$ the present work represents the coupling between the laser cavity and the fiber through a mirror with partial reflectivity, as in the experimental device. The recent dynamical stabilization obtained experimentally by Mitschke and Mollenauer ${ }^{4}$ is mimicked by an overall phase factor in the coupling equations (4.1). The energy loss in the focusing lens is simulated by launching a controlled fraction of the energy into the fiber.

The saturation in the laser is represented through a time-dependent inversion derived directly from the Maxwell-Bloch equations. A further improvement of the synchronized pumped laser model may be obtained by introduction of a time-dependent relaxation inversion, ${ }^{19}$ $\mathscr{D}_{0}(t)$. Such an improvement, however, is not included in the present work.

Finally, the introduction of absorbing boundary layers in the nonlinear Schrödinger equation for the fiber enables us to reduce the pulse spacings, without introducing in- teraction between adjacent pulses, in comparison with the experimental device. In this way a substantial reduction in the number of modes needed for the numerical calculations is achieved.

In the simpler model used by If et $a l .^{7}$ an asymptotically stable state of the widths of the output pulses is not quite obtained after 400 round-trip times, since a slow phase drift could not be eliminated. By the refinements, introduced in the present model, pulse stability is obtained. A pulse compression factor of approximately 10 has been achieved (while only a factor 3 was possible in Ref. 7). However, in the experimental work $^{4}$ a pulse compression factor of 75 was demonstrated. We believe that this level may also be reached in our computational model, if 5-10 times as many cavity modes are included. The corresponding increase of the temporal resolution in the fiber must also be introduced.

\section{ACKNOWLEDGMENTS}

The authors are grateful to L. F. Mollenauer for sending copies of his unpublished papers. J. N. Elgin is thanked for his continued interest in this work. Financial support from the Danish Council for Scientific and Industrial Research is acknowledged.

\section{APPENDIX}

Assume that the solution $E(z, t)$ to Eq. (3.1) only consists of low-amplitude radiation, $\psi(z, t)$ such that the nonlinear term in Eq. (3.1) can be neglected. For simplicity, we let the absorbing function be centered at $t=0$. The equation for $\psi$ then becomes a linear Schrödinger equation with a complex potential, $i \gamma_{0} \operatorname{sech}^{2}(\alpha t)$,

$$
i \psi_{z}+\frac{1}{2} \psi_{t t}+i \gamma_{0} \operatorname{sech}^{2}(\alpha t) \psi=0 \text {. }
$$

For $t \rightarrow-\infty$ the wave is a superposition of incoming and reflected waves, while for $t \rightarrow \infty$ only the transmitted wave exists:

$\psi(z, t)=C e^{i(\omega t-k z)}+D e^{-i(\omega t+k z)}$ for $t \rightarrow-\infty$,

$\psi(z, t)=A e^{i(\omega t-k z)}$ for $t \rightarrow \infty$.

We calculate reflection and transmission coefficients as $R(\omega)=|D / C|^{2}$ and $T(\omega)=|A / C|^{2}$, respectively, with $R+T \leqq 1$ because of the loss.

With $\psi(z, t)=\phi(t) e^{-i k z}$ we obtain from Eq. (A1)

$$
\phi^{\prime \prime}+\alpha^{2}\left[S(S+1) \operatorname{sech}^{2}(\alpha t)-q^{2}\right] \phi=0,
$$

where $S(S+1)=i 2 \gamma_{0} / \alpha^{2}, q=-i \omega / \alpha$, and the frequency is $\omega=\sqrt{2 k}$. Using suitable variable transformations, ${ }^{20}$ Eq. (A3) can be transformed into a hypergeometric differential equation. The asymptotic form of the solution to Eq. (A3) with the representation given by Eq. (A2) becomes

$$
\begin{aligned}
\phi(t) \sim A & \frac{\Gamma(q+1) \Gamma(q)}{\Gamma(q-s) \Gamma(q+S+1)} e^{i \omega t} \\
& \left.+\frac{\Gamma(q+1) \Gamma(-q)}{\Gamma(S+1) \Gamma(-S)} e^{-i \omega t}\right] \text { as } t \rightarrow-\infty,
\end{aligned}
$$




$$
\phi(t) \sim A e^{i \omega t} \text { as } t \rightarrow \infty .
$$

As a result

$$
\begin{aligned}
& R=|\Gamma(q-S) \Gamma(q+S+1)|^{2} \frac{\cos ^{2}\left(\frac{1}{2} \pi \hat{S}\right)}{\pi^{2}}, \\
& T=|\Gamma(q-S) \Gamma(q+S+1)|^{2} \frac{\sinh ^{2}(\pi \omega / \alpha)}{\pi^{2}},
\end{aligned}
$$

where $\widehat{S}=\left(1+i 8 \gamma_{0} / \alpha^{2}\right),{ }^{1 / 2} S=-\frac{1}{2}(1+\widehat{S})$, and $\Gamma$ is the gamma function.

A numerical calculation of the sum

$$
\begin{aligned}
R+T= & \frac{1}{\pi^{2}}|\Gamma(q-s) \Gamma(q+S+1)|^{2} \\
& \times\left[\cos ^{2}\left(\frac{1}{2} \pi \hat{S}\right)+\sinh ^{2}(\pi \omega / \alpha)\right]
\end{aligned}
$$

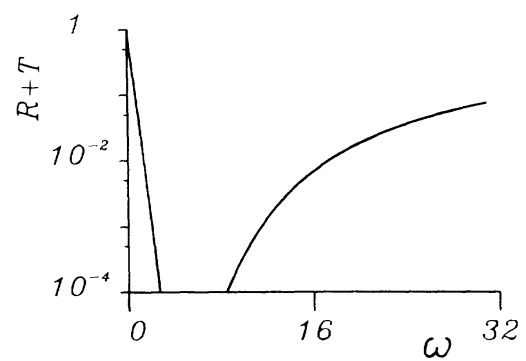

FIG. 10. Scattering from potential $i \gamma_{0} \operatorname{sech}^{2}(\alpha t) . \quad R+T$ given by (A5) vs $\omega$. Parameters: $\gamma_{0}=20, \alpha=1$.

versus $\omega$ is shown in Fig. 10. Since $R+T$ is less than unity (except for $\omega=0$ and $\omega=\infty$ ) the parameters $\gamma_{0}$ and $\alpha$ can be chosen such that (A5) is minimized over a broad region of frequencies.
${ }^{1}$ L. F. Mollenauer, R. H. Stolen, and J. P. Gordon, Phys. Rev. Lett. 45, 1095 (1980).

${ }^{2}$ L. F. Mollenauer and R. H. Stolen, Opt. Lett. 9, 13 (1984).

${ }^{3}$ L. F. Mollenauer, Phil. Trans. R. Soc. Lond., Ser. A 315, 333 (1985).

${ }^{4}$ F. M. Mitschke and L. F. Mollenauer, IEEE J. Quantum Electron. QE-22, 2242 (1986).

${ }^{5}$ L. F. Mollenauer, N. D. Vieira, and L. Szeto, Opt. Lett. 7, 414 (1982).

${ }^{6} \mathrm{H}$. A. Haus and M. N. Islam, IEEE J. Quantum Electron. QE-21, 1172 (1985).

${ }^{7}$ F. If, P. L. Christiansen, J. N. Elgin, J. D. Gibbon, and O. Skovgaard, Opt. Commun. 57, 350 (1986); Physica 23D, 362 (1986).

${ }^{8}$ K. J. Blow and D. Wood, IEEE J. Quantum Electron. QE-22, 1109 (1986).

${ }^{9}$ I. J. Hodgkinson and J. I. Vukusic, Opt. Commun. 24, 133 (1978); Appl. Opt. 17, 1944 (1978).

${ }^{10}$ H. Haken, Light Vol. II: Laser Dynamics (North-Holland,
New York, 1985), p. 98.

${ }^{11}$ G. H. C. New, Rep. Prog. Phys. 46, 877 (1983).

12J. D. Lambert, Computational Methods in Ordinary Differential Equations (Wiley, New York, 1983), p. 217.

${ }^{13}$ A. Hasegawa and F. Tappert, Appl. Phys. Lett. 23, 142 (1973).

${ }^{14}$ V. E. Zakharov and A. B. Shabat, Zh. Eksp. Teor. Fiz. 61, 118 (1971) [Sov. Phys._JETP 34, 62 (1972)].

15J. Satsuma and N. Yajima, Prog. Theor. Phys. 55, Suppl., 284 (1974).

${ }^{16}$ R. H. Hardin and F. Tappert, SIAM (Soc. Ind. Appl. Math.) Rev. 15, 423 (1973).

${ }^{17}$ T. R. Taha and M. J. Ablowitz, J. Comp. Phys. 55, 192 (1984).

${ }^{18}$ F. If, P. Berg, P. L. Christiansen, and O. Skovgaard (unpublished).

${ }^{19}$ J. N. Elgin (private communication).

${ }^{20}$ L. D. Landau and E. M. Lifshitz, Quantum Mechanics, 2nd ed. (Pergamon, London, 1965), p. 79. 